\title{
African genetics for human society
}

We are inviting presubmission enquiries for Articles, Perspectives and Analyses on human, animal, plant and microbial genetics and genomics from Africa, by corresponding authors living and working in African countries, with the aim of publishing an issue of the journal devoted to African genetics.

$\mathrm{P}$ resubmission enquiries made via our online database (http://mts-ng.nature. com/cgi-bin/main.plex) before the end of January 2019 will permit us to advise you on the suitability of proposed articles to be submitted for peer review within the year. Collaborative projects are of course as welcome as those from single labs or institutions, provided the corresponding author is currently working within Africa.

The next International Congress of Human Genetics (http://www.ifhgs.org/) will be held 7-10 March 2021 in Cape Town, South Africa, and it looks as if this will be a great opportunity to emphasize the centrality of African genetics to understanding of the human genome as well as those of many of our agricultural domestications and microbial foes. Importantly, there is much to celebrate right now. With the encouragement and networking generated by the African Society for Human Genetics and the H3Africa initiative, human genetics is flourishing in many places across the continent, including neurogenetics from Mali, psychiatric genetics from Rwanda, cardiovascular genetics from Egypt, malaria genomics from Burkina Faso, hematology from Ghana and Tanzania, cancer genomics from Senegal and Nigeria, and bioinformatics from South Africa-to mention just a few remarkable areas of research excellence highlighted at a recent conference (http://afshgkigali2018.org/).

External solutions for emerging economies often emphasize gadgets and material innovation. For example, research projects often propose innovations in digital communications, portable diagnostic devices, distribution networks, billing systems, banks and storehouses, hospitals and mobile teams, and alternative energy. Of course, these all have their place in sustainable development. However, what we particularly want to incentivize and celebrate here is the capacity building, collaboration and training that result from building and maintaining knowledge and research networks of skilled people. Starting with trained research teams, knowledge can spread via communications from specialist to practitioners, from human geneticist to public health workers, from plant geneticist to seed breeders and farmers.

Genetics, together with the technologies for reading, storing, analyzing and rewriting genomes, provides the framework for multiple solutions to problems of human health and sustainable economic development. Worldwide, trained geneticists are few and far between, but the fundamental skill set is flexible and as universal as the DNA code itself. So, to explain how our discipline builds capacity for developing countries and emerging markets, it is probably useful to show that a small number of shared principles underlying genetic methods have many applications, and each of these subdisciplines has a role in informing the evolution of the other branches of genetics. The same basic set of ideas, traditions and material culture of our discipline is applied to hereditary and complex human diseases, to livestock breeding and animal health, and to crop yield, nutritional improvement and stress resistance.

Enabling researchers to tackle locally urgent research problems helps us all by reframing what a scientific solution looks like. New insights may arise from challenges as simple as adapting diagnostic criteria to local factors: what does Down syndrome look like in this population? What are the main factors in post-traumatic stress disorder in these people? Why does this painkiller not work here? The solutions to problems emerge locally and may look different to what we might expect. But we can expect that at least some will find universal applications.

Published online: 26 October 2018 https://doi.org/10.1038/s41588-018-0277-7 